\title{
INDAGACIÓN DEL AUTORITARISMO EN LA INFANCIA: VINCULACIONES CON LA ORIENTACIÓN DE DOMINANCIA SOCIAL Y LOS VALORES EN NIÑOS Y NIÑAS DE LA CIUDAD DE CÓRDOBA (ARGENTINA)
}

\section{AUTHORITARIANISM'S INQUIRY IN CHILDHOOD: CORRELATIONS WITH SOCIAL DOMINANCE ORIENTATION AND VALUES IN BOYS AND GIRLS FROM CÓRDOBA CITY (ARGENTINA)}

\author{
María Micaela Marasca* ${ }^{*}$ Roxana Marasca ${ }^{* *}$ Y Débora ImHOFF ${ }^{* * *}$
}

\begin{abstract}
*Licenciada en Psicología. Colaboradora Alumna Extensionista en el Programa Infancia y Juventud de la Secretaría de Extensión Universitaria y Ayudante Alumna de la Cátedra de Psicología Evolutiva de la Niñez en la Facultad de Psicología de la Universidad Nacional de Córdoba (UNC).

E-Mail: micamarasca@hotmail.com

**Licenciada en Psicología. Colaboradora Alumna Extensionista en el Programa Infancia y Juventud de la Secretaría de Extensión Universitaria de la Universidad Nacional de Córdoba (UNC).

***Licenciada en Psicología. Docente e investigadora del Equipo de Psicología Política y Becaria del

Consejo Nacional de Investigaciones Científicas y Técnicas (CONICET).

Equipo de Psicología Política del Laboratorio de Psicología Cognitiva de la Facultad de Psicología, Universidad Nacional de Córdoba.

Enfermera Gordillo esquina Enrique Barros, sin número, Ciudad Universitaria.

(5000) Córdoba, República Argentina.
\end{abstract}

\section{RESUMEN}

El estudio que se informa apuntó a analizar las relaciones existentes entre los niveles de $a u$ toritarismo y de orientación de dominancia social y los valores sociales en población infantil. Se trabajó con una muestra no probabilística de tipo accidental de 280 niños de la ciudad de Córdoba, de ambos sexos, que tenían entre 9 y 11 años. Se administró un cuestionario compuesto por tres escalas que indagaban las variables en estudio, previo consentimiento informado de los padres y autorizaciones de los niños y de las autoridades de las instituciones escolares, atendiendo a las variables sociodemográficas de edad y sexo de los participantes.

Se efectuaron análisis uni y bivariados de las variables estudiadas. Los resultados confirman la relación positiva existente entre la Agresión au- toritaria con la Orientación a la dominancia grupal, entre el autoritarismo en sus dos dimensiones (Agresión autoritaria y Sumisión autoritaria) y la Orientación a la dominancia grupal con los valores sociales de Conservación, y entre la Orientación a la dominancia grupal con los valores de Autopromoción. También complementariamente y de acuerdo a lo esperado, se ratificaron relaciones inversas entre la Oposición a la igualdad con los valores de Apertura al cambio y de Autotrascendencia y entre la Orientación a la dominancia grupal, con los valores de Autotrascendencia. No obstante e inesperadamente se evidenció una relación inversa entre la Sumisión autoritaria con la Oposición a la igualdad y con los valores de Apertura al cambio y entre la Oposición a la igualdad con los valores de Conservación. En función de los resultados obtenidos se discute acerca de las particularidades que ad- 
quiere esta articulación a los fines de realizar un aporte a la comprensión de actitudes autoritarias en la niñez, debido a que es una etapa crucial para la adquisición de actitudes que impactan directamente en el comportamiento político y social (Huerta, Bañuelos, Rodríguez, Luz \& Gómez, 2006, en Imhoff \& Brussino, 2010).

Palabras clave: Autoritarismo; Valores sociales; Orientación de dominancia social; Niños; Psicología Política.

\section{ABSTRACT}

This research, developed in the field of Political Psychology, aims to contribute to the understanding of authoritarian attitudes in during childhood, focusing on the relationships with other psychosocial and psycho-political variables such as social values and social dominance orientation.

It's important to study the authoritarianism nowadays, due to the fact that it would obstruct the progress to a new political and economic model that allows the human development and the citizen's participation and involvement, favouring solidarity bonds and social identity (Zaiter, 2002). Thus, the scientific approach of this phenomenon makes its understanding possible and would give tools that can be used for the strengthening of democracy and the acquisition of values and democratic attitudes. Additionally, it's relevant to study the authoritarian attitudes in childhood owing to the fact that is an area of vacancy in the authoritarianism's field because most of the investigations study the authoritarianism in adults or teenagers.

The research aims to analyze the relationships between the authoritarianism's level, the social dominance orientation's level and social values in children's population. This inquiry is a co-relational study in which the sample was selected using a non-random accidental sampling of 280 children from Córdoba city, male and female, from 9 to 11 years old. The sample consists of $52.3 \%$ of girls and a $47.7 \%$ of boys, whereas in relation to the age distribution, there are $27.7 \%$ of 9 year old children, $32.6 \%$ of 10 year old and $39.8 \%$ of 11 year old. The questionnaire was composed of three scales which inquiry into the variables involved and was applied with a previous parents' and head teachers' consent. These scales were: The adaptation of Altemeyer's (2006) Right-Wing Authoritarianism (RWA) Scale to children from Córdoba city by Imhoff and Brussino (2011a); the adaptation of Sidanius and Pratto's (1999) Social Dominance Orientation (SDO) Scale to children's population from Córdoba city by Imhoff and Brussino (2012), and the adaptation of Schwartz and Rubel-Lifschitz's (2009) Portrait Values Questionnaire (PVQ) to children's population from Córdoba city by Imhoff and Brussino (2011b). In addition, socio-demographic variables such as sex and age were asked. Furthermore, the questionnaires were administered orally and individually to each child. Afterwards, univariate and bivariate analysis were performed using the Statistical Package for the Social Sciences (SPSS).

Regarding the results, unvaried analysis showed a higher level of Authoritarian submission than Authoritarian aggression. In relation to social dominance orientation, high scores in Opposition to equality were obtained and a homogeneous distribution in the level of Group based-dominance. Meanwhile, concerning to the children's valorative preference, high scores were found in Self-enhancement, low scores in Self-transcendence and regarding to Conservation and Openness to change, significant differences were not found between high and low levels. Concerning the relationships between the variables, results confirmed a positive relation between Authoritarian aggression with Group based-dominance; between authoritarianism (both dimensions Authoritarian aggression and Authoritarian submission) and group based-dominance with Conservation; and between group based-dominance with Self-enhancement. Moreover, results also demonstrated a negative relationship between Opposition to equality with Openness to change and Self-transcendence; and between Groups based- dominance with Self-transcendence. On the other hand, results revealed a negative relationship between Authoritarian submission with Opposition to equality and Openness to change; and also between Opposition to equality with Conservation values.

According to these results, the peculiarities that acquire this articulation are discussed in order to contribute to the understanding of authoritarian attitudes in childhood. Due to the fact that is a 
crucial stage for the acquisition of attitudes that impact directly in political and social behaviour (Huerta, Bañuelos, Rodríguez, Luz \& Gómez, 2006).

Key words: Authoritarianism; Social values; Social dominance orientation; Children; Political Psychology.

\section{INTRODUCCIÓN}

El trabajo que se informa estuvo basado en un interés por aportar a la comprensión de las actitudes autoritarias, focalizando en las relaciones que éstas presentan con otras variables de corte psicosocial y psicopolítico tales como los valores sociales y la orientación de dominancia social. Actualmente se considera relevante estudiar el autoritarismo, ya que éste obstaculizaría el avance hacia un modelo político y económico que posibilite el desarrollo humano, la participación e involucramiento de los ciudadanos, favoreciendo lazos de solidaridad e identidad social (Zaiter, 2002). Es de pensar que el abordaje de este fenómeno posibilitará su mejor comprensión y otorgará herramientas posibles de ser utilizadas en el marco de intervenciones tendientes al fortalecimiento de la democracia y a la adquisición de valores y actitudes democráticos.

El autoritarismo ha sido un tema de interés y de debate permanente en las Ciencias Sociales durante el Siglo XX. Aún hoy este constructo sigue siendo una preocupación en las sociedades actuales, en tanto siguen vigentes mecanismos psicosociales que reproducen conductas autoritarias, las cuales se han presentado en los últimos años como una temática de relevancia. En este sentido, si bien los pioneros en el estudio de este constructo fueron Adorno, Frenkel-Brunswuik, Levinson y Sanford (1950) desde una perspectiva psicoanalítica, en la actualidad el exponente más importante en el estudio del autoritarismo es Altemeyer (2006), quien desde una perspectiva sociocognitiva reformula el abordaje del fenómeno, contando su teoría con gran apoyo y validez empírica (Cárdenas \& Parra, 2010; Etchezahar, 2011; Imhoff \& Brussino, 2011a). Este autor entiende al autoritarismo del ala de derechas (RWA por sus siglas en inglés) como el producto de la co-variación de tres grupos de actitudes: sumisión autoritaria (refiere a un alto nivel de sometimiento, legitimación y aceptación de las acciones de las autoridades, aún siendo éstas injustas o ilícitas), agresión autoritaria (alude a la tendencia a perjudicar a personas de ciertos grupos, a partir del convencimiento de que ello se encuentra legitimado por el poder establecido) y convencionalismo (entendido como un alto grado de aceptación y adhesión a los normas sociales).

Siguiendo esta línea de trabajo, se considera importante destacar que Altemeyer (1993) denomina su propuesta teórica como Right-Wing Authoritarianism (RWA), es decir, autoritarismo de derechas, conceptualización que se fundamenta en la asunción de los tres grupos de actitudes definidos anteriormente. El calificativo derecha, según el autor, no alude a una orientación o ideología política, sino que es usado en un sentido psicológico y social. En sus palabras: "este autoritarismo es de derechas en el sentido de que se trata de una sumisión a las autoridades establecidas, su objetivo es preservar el orden establecido agrediendo a aquéllos que lo desafían" (Altemeyer, 1993, p. 8). De este modo, sería posible encontrar actitudes autoritarias tanto en aquellas personas que asumen una ideología de derecha como en las que tengan una ideología de izquierda.

Desde esta perspectiva se plantea que las actitudes autoritarias se adquieren en el marco del aprendizaje social, ya que al igual que cualquier otra actitud, su génesis se encuentra íntimamente vinculada con la interacción con los padres, los grupos sociales, los medios de comunicación, instituciones educativas y el aprendizaje que se logre a partir de ellas (Altemeyer, 1988; Del Águila Tejerina, Jiménez Burillo, Luque, Sangrador García \& Vallespín Oña, 2006). A su vez, Altemeyer (1993) destaca el papel primordial que poseen las normas sociales en la configuración 
de las actitudes autoritarias, en tanto considera que las personas tendientes a actuar autoritariamente aprenden las normas sociales que sustentan la agresión contra aquéllos que quebrantan los valores convencionales, siendo éstas relevantes al momento de explicar la agresión autoritaria. Esto se evidencia en el hecho de que los sujetos que puntúan alto en RWA, poseen mayores prejuicios hacia los grupos donde el prejuicio es socialmente más aceptable (Jiménez Burillo, Del Águila Tejerina, Luque, Sangrador García \& Vallespín Oña, 2006).

Otro aporte de esta línea teórica es que en el desarrollo de las actitudes autoritarias parece ser crucial la etapa de la adolescencia. El fundamento que Altemeyer (1988) postula al respecto, corresponde a que en la infancia se observa un mayor grado de sometimiento y conformidad a la autoridad y un bajo nivel de agresión autoritaria. La agresividad para este autor se desarrollaría durante la adolescencia debido a los rasgos que caracterizan a esta etapa, reforzando así el autoritarismo en la dirección tomada desde la infancia o modificando su curso (Roccato, 1997). Sin embargo, aunque Altemeyer centra su enfoque en la adolescencia, otros autores (Boehnke \& Rippl, 1995) indican que en ese período lo que se modifica es el nivel de autoritarismo, mientras que su adquisición sería previa. $\mathrm{Si}$ bien este debate acerca de la etapa en la cual se produce el proceso de adquisición de las actitudes autoritarias continúa vigente, no se puede negar que los individuos se constituyen como seres políticos desde la infancia (Benedicto \& Morán, 1995). En este sentido, es a través del proceso de socialización general de las personas y de la socialización política en particular, que el niño va configurando sus creencias, valores y representaciones acerca de lo político y lo social, adquiriendo determinado tipo de actitudes y comportamientos, produciéndose de este modo el proceso de formación y desarrollo de una identidad psicopolítica.

Así, el trabajo realizado se propuso trabajar con niños, a los fines de aportar elementos para enriquecer esta discusión. Esto resulta a su vez relevante en tanto constituye un área de vacancia dentro del campo de indagación del autoritarismo.

En la revisión realizada se constató que la mayoría de las investigaciones estudian el autoritarismo en adultos, adolescentes y/o jóvenes. Algunos autores (Duriez, Soenens \& Vansteenkiste, 2007; Imhoff \& Brussino, 2011a) plantean que si bien se destaca usualmente la relevancia de estudiar la génesis de las actitudes autoritarias, sólo pocos trabajos empíricos indagaron cómo estas disposiciones se desarrollan en población infantil.

Particularmente, el presente estudio se llevó a cabo con niños de 9 a 11 años. Esta elección se fundamenta en que en este momento del desarrollo se identifican algunos hitos diferenciales, a partir de los cuales de modo progresivo los niños comienzan a tener una conducta más independiente y a desarrollar valores de manera más autónoma, en tanto empiezan a cuestionar las pautas adultas. Si bien su comportamiento no deja de apuntalarse en los modelos incorporados en el ámbito de la familia, en la escuela y en la interacción con pares, a esta edad comienzan a construir sus propias reglas, orientándose preferentemente por determinados valores en detrimento de otros y esta adhesión a su vez se vuelve gradualmente más reflexiva (Arce, Cordera \& Perticarari, 2010).

Igualmente, otros autores (Corona Caraveo \& Fernández Poncela, 2000, en Imhoff $\&$ Brussino, 2010) postulan que a esta edad los niños empiezan a desarrollar el pensamiento abstracto, a partir del cual comienzan a ordenar el mundo político estableciendo relaciones entre los distintos actores que forman parte de ese sistema. Así, comienzan a comprender una terminología política abstracta, apropiándose de ella mediante un pensamiento crítico, con la finalidad de comprender al sistema político en su complejidad. Por ende, es alrededor de los 11 años que los niños ya han alcanzado las capacidades cognitivas asociadas con la formación ciudadana, las cuales harán posible predecir actitudes sociopolíticas futuras (Huerta, Bañuelos, Rodríguez, Luz \& Gómez, 2006).

Por otra parte, RWA ha sido vinculado con otras variables psicosociales y psicopolí- 
ticas que permiten comprender mejor el fenómeno como la orientación de dominancia social (ODS - Cárdenas \& Parra, 2010; Cima \& Dallago, 2007; Duckitt, 2001; Petit \& Costa, 2011) y los valores sociales (D'Adamo \& García Beaudox, 1996; Roccato, Gattino \& Patris, 2000; Seoane \& Garzón, 1992).

La orientación de dominancia social es definida como la "predisposición individual hacia las relaciones intergrupales jerárquicas y no igualitarias" (Petit \& Costa, 2011, p. 73) y alude a la tendencia de las personas a comprender el mundo en términos competitivos entre los grupos sociales, a partir de lo cual de manera visible o no, se conserva la estratificación social, fomentando el dominio de aquellos grupos considerados superiores sobre los inferiores (Petit, López \& Sánchez, 2010). De hecho, los individuos que puntúan alto en ODS manifiestan estar mayormente de acuerdo con medidas duras de control social y con la violencia colectiva (Zubieta, Delfino \& Fernández, 2007).

En esa línea, un estudio realizado en Israel con padres, niños y adolescentes en el cual se aplicó la escala RWA entre otras, se observó que los niños con niveles altos de RWA resultaron ser quienes rechazaban a otros grupos diferentes al que ellos pertenecían, siendo a su vez los que más se asociaban con la conducta de bullying. Así, el autor concluye que los individuos autoritarios poseen una mirada conservadora, son sumisos ante la autoridad y tienen una actitud de rechazo hacia otros grupos (Knafo, 2003). Como vemos, esta orientación psicológica resulta relevante en tanto no sólo permite comprender las diferencias individuales con respecto a las actitudes y conductas sociopolíticas, sino que además posibilita entender cómo se diferencian los grupos sociales en cuanto al favoritismo endogrupal y a la adquisición de nuevos roles colectivos en relación con los distintos niveles de jerarquía (Sidanius, Pratto, Van Laar \& Levin, 2004).

Por otro lado, en lo que refiere a los valores sociales, éstos han sido vinculados con las actitudes autoritarias debido a que ambas ejercen gran influencia en la configuración de la personalidad, en la conducta de los in- dividuos y en sus relaciones interpersonales (Ortiz Zabala, 1985). Los valores sociales son entendidos como "metas deseables, transituacionales, variables en su grado de importancia y que orientan la vida y las conductas de un individuo o de una institución" (Castro Solano \& Nader, 2006, p. 160). Esta conceptualización, proveniente de la teoría de los valores sociales de Schwartz, diferencia un valor de otro en base al tipo de meta motivacional que manifiestan. En el marco de dicha teoría, los valores se agrupan en dos bipolaridades basadas en metas motivacionales diferenciales: Conservación versus Apertura al cambio y Autotrascendencia versus Autopromoción.

En lo que concierne a la relación entre el autoritarismo, la orientación de dominancia social y los valores sociales, estudios efectuados con jóvenes y adultos evidencian que RWA y ODS son constructos que se relacionan de manera positiva entre sí (Cárdenas \& Parra, 2010; Cohrs, Moschner, Maes, Kielmann, 2005; Duriez et al., 2007; Petit \& Costa, 2011; Rottenbacher de Rojas, 2010; Rottenbacher de Rojas, Amaya López, Genna Miyahira \& Pulache Páez, 2009; SilvánFerrero \& Bustillos, 2007; Van Hiel, Pandelaere \& Duriez, 2004; Zubieta et al., 2007). En este sentido, Rottenbacher de Rojas y colaboradores (2009) sugieren que ambas son variables afines a partir de las cuales sería posible predecir la ideología política de las personas. En base a los resultados obtenidos en su estudio, efectuado con adolescentes y habiendo hallado una correlación positiva entre RWA, ODS y la percepción de inseguridad ciudadana, los autores además infieren que una mayor tendencia hacia el autoritarismo estaría asociada con la creencia de que la inseguridad y la delincuencia están vinculadas con aquellos grupos de la sociedad que poseen un menor estatus social. Asimismo, en otro estudio efectuado con jóvenes peruanos, Rottenbacher de Rojas (2010) obtuvo una relación directa entre RWA y ODS, lo que según el autor se asociaría con el hecho de que ambas medidas son expresiones de un conjunto de creencias denominado ideología conservadora. 
Si bien la mayoría de las investigaciones revisadas han obtenido una correlación positiva y fuerte entre RWA y ODS, Duckitt (2001) sostiene que también es posible observar una correlación nula entre estos dos constructos, lo cual estaría asociado básicamente a dos aspectos: por un lado, al grado de contraste ideológico según el eje derecha-izquierda del país en el cual se lleve a cabo el estudio, siendo más alta la relación entre ambos constructos cuanto mayor sea el nivel de contraste ideológico, mientras que en aquellos países poco ideologizados con frecuencia estas dos variables se mostrarían independientes. Por otro lado, el otro aspecto que según Duckitt (2001) condicionaría esta relación es la edad de los entrevistados, correspondiendo a mayor edad una mayor correlación.

Sin embargo, investigaciones más recientes (Cima \& Dallago, 2007; Espinoza, Calderón-Prada, Burga \& Güímac, 2007; SilvánFerrero \& Bustillos, 2007) obtuvieron resultados opuestos con respecto a la hipótesis del contraste ideológico de Duckitt. Por ejemplo, Cima y Dallago (2007) estudiaron la relación entre RWA y ODS en sujetos italianos, cuyo país se caracteriza por un alto nivel de contraste ideológico, obteniendo resultados similares a países poco ideologizados, con lo cual el nivel de ideología sería una variable débil para sustentar la teoría de Duckitt de acuerdo con Cima y Dallago. Aún más, la mayor correlación obtenida en este estudio entre RWA y ODS se observaron en jóvenes, resultados que nuevamente se contraponen a los de Duckitt (Cima \& Dallago, 2007).

Otros estudios (Cárdenas \& Parra, 2010; Pettigrew \& Meertens, 1995; Silván-Ferrero \& Bustillos, 2007; Van Hiel et al., 2004; Zubieta et al., 2007) encontraron que si bien RWA y ODS se relacionan positivamente, son constructos diferentes que se sustentan en mecanismos psicológicos distintos y se asocian a valores sociales también distintos. Por ejemplo, Zubieta y colaboradores (2007) han hallado que mientras RWA hace referencia a la sumisión y agresión motivada por el miedo y la amenaza, ODS se relaciona mayormente con el poder y el prejuicio. En esa misma línea, otros autores
(Silván-Ferrero \& Bustillos, 2007; Van Hiel et al., 2004) postulan que ambas variables conforman distintas medidas del prejuicio. Por un lado, las personas autoritarias tienden a aceptar sumisamente las normas que se les imponen, trayendo esto aparejado un trato indigno hacia el exogrupo y por otro lado, el prejuicio basado en la dominancia busca legitimar la desigualdad que beneficia al propio grupo. Aún más, estudios llevados a cabo en España (Pettigrew \& Meertens, 1995) agregan que RWA se asocia a prejuicios expresados en forma manifiesta, mientras que en relación a ODS el prejuicio se evidencia a través de actitudes más sutiles.

Además es preciso destacar que otras investigaciones (Duriez et al., 2007; Petit \& Costa, 2011) efectuadas con adolescentes, si bien encontraron una correlación positiva entre RWA y ODS, ésta resultó moderada, a diferencia de las analizadas con muestras de adultos. Por ejemplo, Petit y Costa (2011) en su estudio efectuado en Argentina hallaron un bajo nivel de ODS y un bajo nivel de RWA, encontrando que ambas variables se relacionan positivamente aunque en forma moderada. Del mismo modo, Duriez y colaboradores (2007) en su estudio realizado en Bélgica con una muestra de estudiantes secundarios, obtuvieron una relación positiva aunque moderada entre estos dos constructos.

Por otra parte, gran parte de los trabajos revisados hallaron que RWA y ODS son constructos que no sólo se sustentan en mecanismos psicológicos distintos, sino que además se relacionan con valores sociales diferentes. Así, se observa que RWA se relaciona fuerte y positivamente con los valores que conforman la sub-dimensión Conservación (tradición, conformidad y seguridad) y débil y negativamente con la sub-dimensión Apertura al cambio, fundamentalmente con los valores autodirección y estimulación (Giovanni Di Stefano, 2005; Van Hiel et al., 2004), así como también con la sub-dimensión Autotrascendencia, especialmente con sus valores universalismo y benevolencia (Cohrs et al., 2005; Giovanni Di Stefano, 2005).

Por otro lado, hay evidencias de una correlación fuerte y positiva entre ODS y los va- 
lores poder y logro que conforman la sub-dimensión Autopromoción (Altemeyer, 1998, 2004; Espinoza \& Calderón-Prada, 2006; Giovanni Di Stefano, 2005; Mendoza, 2010; Van Hiel et al., 2004; Zubieta et al., 2007; Zubieta \& Delfino, 2011) y de una relación baja y negativa de ODS con la sub-dimensión Autotrascendencia, conformada por los valores universalismo y benevolencia (Altemeyer, 1998, 2004; Cohrs et al., 2005; Espinoza \& Calderón-Prada, 2006; Giovanni Di Stefano, 2005; Mendoza, 2010; Van Hiel et al., 2004). En la misma línea, el estudio de Roccato y colaboradores (2000), efectuado en Turín con universitarios, encontró que mientras RWA se relaciona predominantemente con valores tradicionales, ODS se relaciona positivamente con valores relativos a la auto-realización. Sin embargo, otros estudios (Altemeyer, 2006; Roccato et al., 2000; Rottenbacher de Rojas, 2010) también han evidenciado que RWA no sólo se relaciona con valores tradicionales, sino también con los valores seguridad, conformismo y benevolencia (Altemeyer, 2006). A partir de estos resultados se infiere que los individuos que puntúan alto en la escala RWA, tienden a establecer y mantener el orden, el control y la estabilidad social (Rottenbacher de Rojas, 2010).

En lo que respecta a Latinoamérica en particular, el estudio de Mendoza (2010) 1levado a cabo con habitantes de distintos países latinoamericanos y de la Península Ibérica obtuvo como resultado que Argentina, México y Brasil adhieren en mayor medida al autoritarismo en comparación con la Península Ibérica y Chile, en tanto obtuvieron puntuaciones más elevadas tanto para la escala total como para cada sub-dimensión. No obstante, y contrariamente a lo esperado, no se hallaron diferencias estadísticamente significativas entre Argentina, México, Brasil, Chile y la Península Ibérica en ODS, ya que las puntuaciones obtenidas para este constructo fueron semejantes. Asimismo, se halló que RWA correlaciona fuerte y positivamente con la Conservación (tradición, conformidad y seguridad), mientras que ODS se relaciona positivamente y en mayor medida con la Autopromoción (poder y logro). A su vez, se ratifica que ambos constructos correlacionan negativamenre con la Autotrascendencia (benevolencia y universalismo).

Estos resultados fueron reforzados por otro estudio (Zubieta et al., 2007) realizado en Argentina con estudiantes universitarios, en el cual se obtuvo como resultado una mayor preferencia por valores de Autotrascendencia y Apertura al cambio, mostrando un bajo nivel de ODS. Además, se observó que ODS se relaciona de forma positiva con la Autopromoción y negativamente con la Autotrascendencia. Esto evidenciaría, según las autoras, que las personas con alto nivel de ODS y bajas puntuaciones en valores de Autotrascendencia, poseen una falta de interés por el bienestar de los demás, centrándose más en las metas individuales y en el beneficio propio.

Así, a partir de estos antecedentes se observa la relevancia de abordar el análisis de las actitudes autoritarias en la infancia, en tanto constituye un área de vacancia dentro del campo de indagación del autoritarismo. A su vez, se considera pertinente complejizar el análisis del fenómeno a través de la inclusión de otras variables psicosociales y psicopolíticas, en las que la información sobre niños también es escasa. En este sentido, el objetivo del trabajo realizado fue analizar el nivel de autoritarismo de niños y niñas de 9, 10 y 11 años de la ciudad de Córdoba, en relación con su nivel de orientación de dominancia social y el tipo de valores sociales que ellos poseen.

\section{Metodología}

A los fines de alcanzar los objetivos propuestos se eligió un esquema de investigación de tipo correlacional (Hernández Sampieri, Fernández Collado \& Baptista Lucio, 2003).

\section{MUESTRA}

Se trabajó con una muestra no probabilística accidental constituida por 280 niños de la ciudad de Córdoba, de ambos sexos, 
con edades comprendidas entre los 9 y 11 años, que asistían al nivel primario de escuelas públicas y privadas.

Inicialmente, se administraron las encuestas a 312 niños. Sin embargo, se detectó que durante la aplicación del instrumento algunos participantes se cansaban y no llegaban a responder todos los ítemes, por lo cual se decidió conservar sólo los casos en los que sí se respondieron las tres escalas. Así, la muestra quedó compuesta por 280 sujetos $(52.3 \%$ niñas y $47.7 \%$, niños). Las edades se repartieron en un $27.6 \%$ niños y niñas de 9 años, un $32.6 \%$ de 10 años y un $39.8 \%$ de 11 años, con una media igual a 10.12 años y una desviación estándar igual a .8 .

Cabe señalar que si bien la muestra seleccionada estuvo conformada por alumnos pertenecientes a instituciones educativas tanto públicas como privadas, en esta ocasión se decidió no tomar en cuenta el nivel socioeconómico y cultural de la población, debido a dificultades en el trabajo de campo que impidieron el análisis de esta variable. No obstante, se considera que su análisis hubiera resultado muy interesante y hubiera generado un valioso aporte, por lo que no se descarta su inclusión en futuras investigaciones.

\section{INSTRUMENTOS}

Se administraron los siguientes instrumentos:

- La adaptación idiomática y cultural al contexto local (Córdoba, Argentina) de Imhoff y Brussino (2011a) de la Escala de Right-Wing Authoritarianism (RWA) de Altemeyer (2006). Según Altemeyer, esta escala recupera los componentes principales del autoritarismo de derechas en dos sub-dimensiones: Agresión autoritaria y Sumisión autoritaria. Está compuesta por 12 ítemes, 7 de los cuales corresponden a Agresión autoritaria, mientras que los 5 restantes conforman la subdimensión Sumisión autoritaria. Posee una opción de respuesta situada en una escala Likert de 3 puntos, en la que 1 in- dica No estoy de acuerdo y 3: Estoy muy de acuerdo. Según las autoras (Imhoff \& Brussino, 2011a), la escala evidencia índices de confiabilidad satisfac- torios en población infantil $(\alpha=.69$ para la sub-escala de Agresión autoritaria y $\alpha=.63$ para la sub-escala de Sumisión autoritaria), lo cual coincide con lo que postulan otros autores (Lemos, 2006; Mischel, Zeiss, R. $\&$ Zeiss, A., 1974), quienes señalan que índices de fiabilidad de .30 o superiores suelen ser considerados como significativos cuando se trabaja con muestras infantiles, dado que las respuestas de los niños se caracterizan por ser todavía inestables.

- La adaptación a población infantil cordobesa de Imhoff y Brussino (2012) de la Escala de Orientación de Dominancia Social (ODS) de Sidanius y Pratto (1999) que evalúa el grado en que las personas sostienen un esquema referencial basado en la desigualdad entre los grupos y en diversas formas de desigualdad social. Está compuesta por 10 ítemes en los cuales los participantes indican en qué medida están de acuerdo con cada afirmación en base a una escala Likert de 3 puntos (1: No estoy de acuerdo a 3: Estoy muy de acuerdo). En el estudio con población infantil cordobesa se corroboró la estructura bidimensional de la escala. Así, se evidencia una dimensión vinculada con la Oposición a la igualdad compuesta por 5 ítemes $(\alpha=.62)$ y otra dimensión asociada con la Orientación a la dominancia grupal también con 5 ítemes $(\alpha=.62)$.

- La adaptación al idioma español y para población infantil de Imhoff y Brussino (2011b) del Portrait Values Questionnaire (PVQ) de Schwartz y Rubel-Lifschitz (2009). Este cuestionario está constituido por 16 ítemes que los entrevistados deben responder en una escala tipo Likert de 3 puntos de acuerdo al grado en el que se sienten identificados en cada uno de ellos (1: No es nada parecida/o a mí a 3: Es muy parecida/o a $\mathrm{mi}$ ). Las frases están redac- 
tadas en relación con el sexo del participante. El instrumento indaga los 10 valores propuestos por Schwartz y RubelLifschitz (2009), los cuales se agrupan en cuatro dimensiones: Apertura al cambio versus Conservación y Autopromoción versus Autotrascendencia. De acuerdo al estudio realizado por Imhoff y Brussino (2011b) con población infantil cordobesa se ratificó la estructura teórica propuesta por Schwartz y Rubel-Lifschitz (2009), con la presencia de cuatro factores que explicaron un $40 \%$ de la variancia. Los índices de confiabilidad fueron bajos aunque adecuados para población infantil: $\alpha=.47$ para la dimensión Conservación; $\alpha=.49$ para la dimensión $\mathrm{Au}-$ totrascendencia; $\alpha=.50$ para la dimensión Autopromoción y $\alpha=.41$ para la dimensión Apertura al cambio.

- Se realizaron preguntas cerradas de alternativa fija para evaluar las variables socio-demográficas (edad y sexo de los entrevistados) y también se solicitó el consentimiento informado por escrito a los padres o tutores de los niños para que ellos pudieran participar del estudio.

\section{PROCEDIMIENTO}

La instancia de recolección de datos fue efectuada en el transcurso del año 2011 en escuelas públicas y privadas de nivel primario de la ciudad de Córdoba (Argentina). En primer lugar, se solicitó autorización a las directoras para ingresar a la institución educativa, explicando los fines académicos del trabajo a realizar. Los niños y docentes fueron informados acerca del motivo de la tarea a realizar aclarando que su participación en la misma era de carácter voluntario, confidencial y anónimo. Luego de obtenidos los consentimientos informados se administraron individualmente los instrumentos. Cabe destacar que, antes de comenzar se buscó establecer cierta empatía con el encuestado, con el objetivo de hacer el proceso más ame- no y que el niño pudiera expresar sus dudas y sensaciones a medida que la entrevistadora realizaba la encuesta.

A su vez, se les explicó brevemente a los niños cómo era la modalidad de respuesta establecida para cada una de las tres escalas, mostrándoles las opciones de respuesta en una hoja aparte que podían tener en sus manos para una mejor visualización. También se les mencionó que podían preguntar cualquier duda en relación con los términos que aparecieran en el cuestionario y que no conocieran o que fueran incomprensibles para ellos. Además, es preciso destacar que se respetaron los tiempos de los niños al momento de contestar el cuestionario, así como también sus recreos, instancias de evaluación y cualquier otra eventualidad que hubiera surgido en el momento de su administración.

El cuestionario se administró de forma oral, lo que facilitó la detección de aquellos casos en los que los niños no comprendían algún ítem o se mostraban cansados al momento de brindar su respuesta. Ante este tipo de situaciones, se apeló a explicar el ítem nuevamente o hacer una pausa con los niños que se encontraban fatigados. No obstante, cabe señalar que se decidió contar como parte de la muestra sólo con aquellos niños que lograron responder las tres escalas.

\section{PrepaRACIÓN Y ANÁLISIS DE LOS DATOS}

En primer lugar, se procedió a invertir los ítemes negativos de las escalas RWA y ODS. La escala de valores sociales no presenta ítemes inversos.

Luego se analizó el patrón de valores perdidos. Dado que ningún ítem presentaba más de un $5 \%$ de valores perdidos, se procedió a completar la información faltante mediante el método estimación - maximación (EM), por considerarse uno de los que ofrece una estimación más precisa y razonable efectuada en base a estimaciones de máxima verosimilitud (Hair, Anderson, Tatham \& Black, 1999). Finalmente, se conformaron las subescalas correspondientes a los instrumentos utilizados. 
Se efectuaron análisis bivariados para conocer la relación entre el nivel de RWA con el nivel de ODS, analizar la relación de las dos variables recién mencionadas con los valores sociales e identificar si existen relaciones significativas entre las variables en estudio y las variables edad y sexo de los niños. A tales efectos se calcularon los coeficientes y medidas de correlación y asociación pertinentes, con su correspondiente significación estadística.

\section{Resultados}

Un primer dato de interés, antes de reportar las relaciones entre las variables, es el que concierne a la variable dependiente $\mathrm{Au}$ toritarismo, operacionalizada a través de dos sub-dimensiones: Agresión autoritaria y $\mathrm{Su}-$ misión autoritaria. La primera de ellas posee una amplitud de 7 a 21 puntos (aunque en la muestra estudiada el valor mínimo evidenciado es 8), obteniendo una media igual a 15.52 , una mediana igual a 16 y una desviación estándar igual a 2.973. La mayor cantidad de los casos se concentran principalmente en los puntajes medios de la escala, tratándose de una distribución levemente asimétrica (-.345). La segunda tiene una amplitud de 5 a 15 puntos (aunque en esta muestra el valor mínimo mostrado es 6), una media igula a 13.21, una mediana igual a 14 y una desviación estándar igual a 1.777. Se evidencia una concentración de la mayor parte de los casos en los valores más altos de la variable, lo cual también se hace claro en el mayor valor del índice de asimetría (-1.292).

Con respecto a las frecuencias, y a los fines de conocer la mayor o menor presencia de los constructos, se consideró (a partir de calcular las puntuaciones $Z$ para los valores originales de cada sub-escala) el porcentaje de casos ubicados por debajo de -1 puntuación $Z$ y por encima de +1 puntuación $Z$ como niveles bajos y altos, respectivamente. Así, en Agresión autoritaria, un 19\% de los casos presenta niveles bajos de presencia del atributo, mientras que un $17.9 \%$ evidencia niveles altos de Agresión autoritaria. Respecto de
Sumisión autoritaria, el 16.1\% de los casos se posiciona en un nivel bajo y un $28.3 \%$ manifiesta niveles altos de sumisión. Estos datos evidencian una mayor presencia de sumisión que de agresión autoritaria en los niños.

En un segundo momento se analizaron las relaciones entre las variables psicosociales y psicopolíticas del estudio, las cuales se presentan en la Tabla 1.

En cuanto a las correlaciones que poseen significación estadística, se observa una relación débil y en la dirección esperada entre Agresión autoritaria y la sub-dimensión Orientación a la dominancia grupal. Por otra parte, en lo que concierne a la relación entre la Sumisión autoritaria y la sub-dimensión Oposición a la igualdad de la Orientación a la dominancia social, también se trata de una relación débil aunque en una dirección inesperada, ya que se espera que a mayor Sumisión autoritaria corresponda mayor Oposición a la igualdad.

Por otra parte, en lo vinculado a la relación entre la Agresión autoritaria con la dimensión Conservación de los valores sociales, vemos que se trata de una relación débil. Esto evidenciaría que los niños que tienden a preferir valores conservadores, mostrarían mayores índices de agresión autoritaria que quienes no prefieren estos valores. Además, en lo referente a la relación entre la Sumisión autoritaria con la dimensión Conservación de los valores sociales, se obtuvo una relación moderada y en un sentido positivo. De ahí que los niños que adhieren a valores más conservadores, tenderían a ser más sumisos ante la autoridad.

A su vez, la relación entre Sumisión autoritaria y la dimensión Apertura al cambio, resultó ser débil y en una dirección no esperada, en tanto se espera que los niños con mayor nivel de Sumisión autoritaria estén menos orientados a valores que componen la sub-dimensión Apertura al cambio.

Complementariamente, se indagaron las relaciones entre la Orientación a la dominancia social y los valores sociales, a los fines de complejizar el análisis y se observa que casi todas las correlaciones son significativas estadísticamente (ver Tabla 2). 
Así, en lo concerniente a la relación entre la Oposición a la igualdad con la dimensión Conservación de los valores sociales, se encontró que se trata de una relación moderada, aunque no en la dirección esperada, ya que se esperaba que a mayor Oposición a la igualdad, mayor preferencia por valores de Conservación.

En función de ello, se efectuaron posteriores análisis a los fines de explorar con mayor profundidad dicha correlación. En este sentido, se procedió a analizar la relación entre la Oposición a la igualdad y cada uno de los valores que forman parte de la sub-dimensión de Conservación. Los resultados indican una relación moderada entre Oposición a la igualdad y el valor de conformidad $\left(-.219^{*}\right)$, pero una relación muy baja entre Oposición a la igualdad y el valor de seguridad (-.125*), al mismo tiempo que no se observa una relación atendible ni significativa entre Oposición a la igualdad y el valor de tradición (-.111).

A su vez, se analizó la relación entre Oposición a la igualdad y la sub-dimensión Conservación por la edad de los participantes, a partir de la cual se corroboraron matices diferenciales en función de los distintos grupos etarios. Así, los resultados indican que los niños de menor edad (9 años) evidencian una relación más fuerte entre estos dos constructos $\left(-.30^{*}\right)$, a diferencia de los de $10(-.21 *)$ y $11(-.24 *)$ años. Finalmente, se analizó la relación en función de la variable sociodemográfica sexo de los participantes. En esa línea, la vinculación entre Oposición a la igualdad y Conservación se muestra más fuerte entre los varones $(-.27 * *)$ que entre las niñas $\left(-.18^{*}\right)$.

Con respecto a la relación entre la Oposición a la igualdad y la dimensión Apertura al cambio, se trata de una relación débil y en una dirección esperada. Dichos resultados indicarían que los niños que puntúan alto en la sub-dimensión Oposición a la igualdad, se muestran poco orientados a elegir valores de Apertura al cambio. Por otro lado, en lo que respecta a la relación entre la dimensión de los valores sociales Autotrascendencia y la Oposición a la igualdad se observa una re- lación moderada y en la dirección esperada. Es decir, los niños que se oponen a que todos sean iguales y que tengan las mismas posibilidades, puntuarían bajo en valores como benevolencia y universalismo. En cuanto a la relación entre la Orientación a la dominancia grupal con la dimensión Conservación de los valores sociales, se obtuvo una relación débil en la dirección esperada, lo que señalaría que los niños que poseen mayores índices de Orientación a la dominancia grupal, tenderían a mostrar una mayor adherencia a valores conservadores. Por otra parte, se encontró una relación débil y en dirección esperada entre la Orientación a la dominancia grupal y la dimensión de los valores sociales Autotrascendencia. Esto mostraría una tendencia en el sentido de que aquellos niños que tienden a preocuparse por los demás y a preferir valores como benevolencia y universalismo, mostrarían bajos niveles de Orientación a la dominancia grupal. Igualmente, se halló una relación moderada y positiva entre la Orientación a la dominancia grupal y la dimensión Autopromoción.

En lo que se refiere a las distintas variables en estudio y su relación con la edad, los resultados mostraron que entre Agresión autoritaria y la edad de los niños se observa una relación débil, con significación estadística y en dirección inversa $(r=-.108 ; p=.036)$. Esto indicaría que inesperadamente según los antecedentes teóricos revisados, los niños a medida que crecen tenderían a disminuir sus niveles de Agresión autoritaria. Por su parte, entre Sumisión autoritaria y la edad de los participantes, no se halló una relación atendible $(r=-.019 ; p=.377)$.

Con respecto a la correlación entre la subdimensión Oposición a la igualdad con la edad de los niños se encontró una relación débil y en una dirección no esperada aunque estadísticamente significativa $(r=-.207$; $p=.000)$, en tanto es esperable que a mayor edad, los niños posean mayores niveles de Oposición a la igualdad. Asimismo, se halló una relación débil, estadísticamente significativa aunque en dirección no esperada $(r=-.152 ; p=.005)$ entre la sub-dimensión 
Orientación a la dominancia grupal y la edad, ya que según los antecedentes sería esperable que a medida que los niños van creciendo, aumente su nivel de Orientación a la dominancia grupal.

Por su parte, la sub-dimensión Conservación de los valores sociales con la edad de los participantes mostró una correlación prácticamente nula, sin significación estadística $(r=-.028 ; p=.320)$. Del mismo modo, la correlación entre la dimensión Apertura al cambio de los valores sociales con la variable sociodemográfica edad fue prácticamente nula $(r=.066 ; p=.137)$. Por otro lado, en la correlación de la dimensión Autotrascendencia de los valores sociales con la edad se halló una relación débil aunque estadísticamente significativa y en una dirección positiva $(r=.102 ; p=.044)$. Es decir que los niños a medida que crecen tenderían a priorizar valores como universalismo y benevolencia. Por último, en lo que concierne a la correlación entre la dimensión Autopromoción de los valores sociales con la variable sociodemográfica edad de los participantes se encontró una relación nula y no significativa estadísticamente $(r=.002 ; p=.488)$.

Por otro lado, en lo que respecta a la asociación entre las distintas variables del estudio realizado con el sexo de los participantes, ninguna de las vinculaciones resultó estadísticamente significativa.

Como se observa, muchos de los resultados encontrados no van en la dirección esperada, lo cual posiblemente podría estar asociado con el hecho de que estas actitudes están desarrollándose en esta etapa y todavía no se encuentran consolidadas. Además, tal como plantea la perspectiva cognitivoevolutiva, el desarrollo que va realizando el niño en la comprensión del mundo social, no sólo depende de la calidad y tipo de experiencias sociales que va entablando con otras personas o agencias socializadoras, sino que el factor intelectual también resulta determinante (Enesco, Delval, Navarro, Villuendas, Sierra \& Penarda, 1995). Por lo que si se habla en términos del desarrollo del pensamiento y de la moralidad, un niño de 9 años difiere de uno de 11 años.

\section{Discusión}

La presencia de las relaciones sociales en las que aparece el autoritarismo y en las que prevalece la imposición de poder de unos sobre otros se manifiestan como una realidad constante en nuestros días. Este tipo de actitudes impregna la interacción entre los individuos tanto a nivel interpersonal como intra e intergrupal, lo que dificulta y vulnera las prácticas que apuntan a fortalecer la democracia (Zaiter, 2002). Es en torno a este tipo de cuestionamientos que el estudio realizado estuvo basado en un interés por aportar a la comprensión de las actitudes autoritarias en la infancia, en tanto es una etapa clave para la adquisición y aprendizaje de actitudes que impactan en el comportamiento social y político (Huerta et al., 2006, en Imhoff \& Brussino, 2010). Así, se indagó la presencia de este tipo de actitudes en una población infantil, profundizando el análisis a través de explorar sus relaciones con otras variables psicosociales y psicopolíticas.

Además, se analizaron los niveles de autoritarismo que presentaban los niños de la muestra. Así, se encontró un mayor nivel de Sumisión autoritaria que de Agresión autoritaria, lo que coincide con la propuesta de Altemeyer (1988) según la cual en la infancia los niños tienden a aceptar las normas y a no contradecir la autoridad, siendo recién en la adolescencia cuando empiezan a desarrollar un mayor nivel de Agresión autoritaria debido a los cambios característicos de esa etapa. Estos resultados también se corresponden con lo hallado por Imhoff y Brussino (2011a). Las autoras asocian la mayor presencia de Sumisión autoritaria en esta etapa con un modelo adultocéntrico a partir del cual el adulto se vincula con el niño pensándolo como un ser pasivo, incapaz y sumiso ante la autoridad. Es decir, teniendo como meta principal la adaptación de los niños al contexto y no la problematización y el desarrollo de un espíritu crítico por parte de ellos. En relación con esto, acordamos con las autoras en que es preciso reflexionar acerca de los paradigmas de infancia existentes en nuestra cultura, repen- 
sando nuestra concepción de la niñez. Por ello, queda planteada la necesidad de generar propuestas concretas de intervención buscando un cambio de la perspectiva cultural en la que los niños puedan ser escuchados y su opinión valorada, a los fines de lograr una participación activa y protagónica en su desarrollo y en el de su sociedad.

Por otra parte, si bien se observó un menor porcentaje de Agresión autoritaria que de Sumisión autoritaria, esto no significa la ausencia de la primera, lo que permitiría pensar en la presencia de los dos componentes del autoritarismo en la niñez, en contraposición con la propuesta de Altemeyer (1988) y coincidiendo con Boehnke y Rippl (1995), quienes indican que en la adolescencia lo que se modifica es el nivel de autoritarismo y que su adquisición sería previa. No obstante, creemos que los resultados obtenidos no proporcionan evidencia empírica suficiente para resolver esta discusión acerca del período evolutivo en el que se adquiere este tipo de actitudes. Para ello, sería preciso un estudio longitudinal (Delgado Rodríguez \& Llorca Díaz, 2004) en el que se pueda corroborar y obtener mayor información acerca de la etapa en la que se adquieren las actitudes autoritarias y observar si los niveles de agresión y sumisión autoritaria se modifican a lo largo del ciclo vital.

Aún así, no se puede negar que ya durante la niñez los individuos se constituyen como seres políticos, siendo a través del proceso de socialización que el niño va configurando sus creencias, valores y representaciones acerca de lo político y lo social, además de adquirir determinado tipo de actitudes y comportamientos, produciéndose así el desarrollo de una identidad psicopolítica (Benedicto \& Morán, 1995). Por ello, se destaca la relevancia de estudiar este tipo de fenómeno en la infancia debido a que, como plantea Knafo (2003), los niños con alto nivel de autoritarismo resultaron ser quienes rechazaban a otros grupos diferentes a los que ellos pertenecían y los que más se asociaban con la conducta de bullying.

Esto invita a pensar acerca de la importancia de otra variable psicosocial clave: la
Orientación a la dominancia social. Al respecto se encuentra que la Agresión autoritaria se vincula con dicha variable, en el sentido de que a mayor presencia de un atributo, mayor presencia del otro. Estos resultados coinciden con la mayoría de los estudios revisados (Cárdenas, Meza, Lagues \& Yañez, 2010; Cárdenas \& Parra, 2010; Chatard \& Selimbegovic, 2008; Cima \& Dallago, 2007; Duckit, 2001; Duriez et al., 2007; Espinoza et al., 2007; García-Castro, 2010; Lacerda Teixeira Pires, 2010; Montes Berges \& Rodríguez García, 2006; Petit \& Costa, 2011; Petit et al., 2010; Roccato et al., 2000; Rottenbacher de Rojas, 2010; Rottenbacher de Rojas et al., 2009; Silván-Ferrero \& Busti1los, 2007; Zubieta et al., 2007), cuyos autores también han obtenido una correlación directa y significativa entre ambos constructos, aludiendo a que se trata de variables afines a partir de las que sería posible predecir la ideología política de las personas (Rottenbacher de Rojas et al., 2009).

Sin embargo, y a diferencia de la mayor parte de los antecedentes anteriormente reseñados, que evidenciaron una relación fuerte entre ambas variables, la correlación hallada en el estudio realizado resultó débil. No obstante, es de destacar que otras investigacones (Duriez et al., 2007; Petit \& Costa, 2011), cuyas muestras estuvieron compuestas por adolescentes y jóvenes, encontraron una relación moderada entre ambas variables, siendo estos resultados más semejantes a los obtenidos en este trabajo. A partir de esto, se podría pensar en la existencia de diferencias en la intensidad de la relación entre dichas variables en función de la edad de los participantes. Quizás, al avanzar la maduración, la relación entre estas variables resulte más estable, por lo que se considera que sería interesante analizar este supuesto a través de estudios longitudinales.

A su vez, se halló que la Sumisión autoritaria se relaciona inversamente con la Oposición a la igualdad, lo que contradice lo hallado por los antecedentes recuperados. No obstante, se podría pensar que tal vez el hecho de que los niños que se muestran sumisos frente a la autoridad, sean también 
quienes se muestran a favor de la igualdad, podría asociarse con cierta deseabilidad social. Sería preciso llevar a cabo otros estudios a los fines de corroborar esta hipótesis.

En lo que respecta a la correlación entre el Autoritarismo y los valores sociales, se encontró que tanto la Agresión autoritaria como la Sumisión autoritaria parecen apoyarse en los valores de Conservación. Así, una alta puntuación en cualquiera de las dos sub-dimensiones de Autoritarismo va acompañada de una puntuación elevada en la variable que refiere a los valores de conformidad, tradición y seguridad. Estos hallazgos se corresponden con la mayoría de los antecedentes reseñados (Altemeyer, 1998, 2006; Cohrs et al., 2005; Giovanni Di Stefano, 2005; Mendoza, 2010; Rocatto et al., 2000; Rottenbacher de Rojas, 2010; Van Hiel et al., 2004; Zubieta \& Delfino, 2011).

Por otro lado, y contrariamente a lo esperado, se obtuvo que la Sumisión autoritaria parece relacionarse también, de forma directa, con los valores autodirección y estimulación, lo cual se contrapone a los hallazgos obtenidos por estudios previos (Altemeyer, 1998; Giovanni Di Stefano, 2005; Van Hiel et al., 2004). Esto podría vincularse con el hecho de que aún la personalidad de los niños no está completamente formada y por tanto no tienen una adhesión estable a los valores, como resulta al aplicar esta escala en población adulta.

En lo que refiere a la correlación entre ODS y los valores sociales, se encontró tal como se esperaba, que la Oposición a la igualdad se relaciona negativamente con los valores de la sub-dimensión Apertura al cambio, lo cual coincide con los hallazgos obtenidos por Zubieta y colaboradores (2007). Estos resultados quizás sugieran que los niños que se oponen a la igualdad tienden a priorizar en menor medida valores tales como la autodirección y la estimulación, poniendo por tanto menor énfasis en la acción y pensamiento independientes y en la búsqueda de nuevos desafíos en la vida.

Asimismo, en el estudio realizado se encontró que ambas sub-dimensiones de la
ODS (Oposición a la igualdad y Orientación a la dominancia grupal) correlacionan de manera inversa y esperada con la Autotrascendencia, resultados que refuerzan hallazgos obtenidos por estudios previos (Altemeyer, 1998, 2006; Espinoza \& CalderónPrada, 2006; Mendoza, 2010; Van Hiel et al., 2004; Zubieta et al., 2007). Así pues, los niños que puntúan bajo en Orientación a la dominancia social, prefieren valores orientados al bien común tales como el universalismo y la benevolencia. En este sentido, se podría pensar que en la medida en que se lleven a cabo intervenciones tendientes a desarrollar y reforzar valores de índole colectiva, quizás se lograrían atenuar aquellas ideologías y creencias que se oponen a la igualdad y que tienden a dominar a otros.

También se encontró como se esperaba, que la Orientación a la dominancia grupal se relaciona positivamente con valores que componen la sub-dimensión Autopromoción. Estos datos ratifican los resultados obtenidos por la mayoría de los estudios previamente reseñados (Altemeyer, 1998; 2006; Cohrs et al., 2005; Espinoza \& CalderónPrada, 2006; Mendoza, 2010; Van Hiel et al., 2004; Zubieta et al., 2007; Zubieta \& Delfino, 2011) y confirman otra de las hipótesis de este trabajo, que proponía que los niños que puntuaran alto en ODS, estarían mayormente orientados a preferir valores individualistas, como el poder y el logro, en desmedro de valores colectivistas. Así, los hallazgos obtenidos reforzarían la idea de Llinares Insa, Molpeceres y Musitu (2001) que plantea que mientras los niños estén orientados a preferir valores en los que se priorice la autorrealización y el éxito personal, mostrarán una mayor ODS. A partir de estos resultados, se proponen líneas de intervención tendientes a desalentar este tipo de actitudes y a reforzar aquellos valores orientados al bien común, ya que tal como postula Schwartz y Rubel-Lifschitz (2009), las preferencias de valores y los sistemas de creencias de las personas tienen gran incidencia en su comportamiento, en las relaciones interpersonales actuales y futuras y en la configuración de la personalidad. 
Además, se obtuvo una relación positiva entre la Orientación a la dominancia grupal y la sub-dimensión Conservación, lo cual también ha sido corroborado por Zubieta y Delfino (2011) en su estudio. Dicha relación es esperable, ya que tanto la Orientación a la dominancia social como el Autoritarismo se encuentran enmarcadas dentro de aquellas variables que expresan una ideología política conservadora (Rottenbacher de Rojas, 2010). Estos resultados quizás podrían indicar que los niños que tienden a dominar a otros y a expresar su rechazo hacia grupos de menor estatus, serían quienes enfatizan el respeto por costumbres e ideas tradicionales y tenderían a buscar la estabilidad del orden social, de las relaciones y de sí mismos. De este modo, como plantea Rottenbacher de Rojas (2010), se cree que esta motivación de superioridad grupal sentaría las bases para la formación de prejuicios, el mantenimiento de las jerarquías sociales y el conservadurismo.

En lo concerniente a la relación entre la Oposición a la igualdad con la sub-dimensión Conservación de los valores sociales se encontró una correlación inversa e inesperada, resultados que contradicen lo hallado por lo previamente reseñado (Zubieta \& Delfino, 2011), en tanto es esperable que a mayor Oposición a la igualdad resulte una mayor preferencia por valores de Conservación. No obstante, como se mencionó con anterioridad, cuando se indagó en la relación entre la Oposición a la igualdad con cada uno de los valores que conforman la sub-dimensión de Conservación (conformidad, seguridad y tradición), se encontró que si bien las relaciones continuaban siendo negativas y, por tanto no esperadas, el valor de conformidad fue el que evidenció una correlación más alta y mayormente significativa, en comparación con los valores de seguridad y tradición. Así, estos resultados demuestran que quizás no sea la sub-dimensión Conservación en su totalidad la que se vincula con Oposición a la igualdad, sino que parece ser una relación más específica, en la que sería necesario profundizar en futuras investigaciones.

A su vez, se corroboraron matices diferenciales en la relación entre la Oposición a la igualdad y la sub-dimensión Conservación en función de la edad de los participantes, encontrando que son los niños de menor edad (9 años) los que evidencian una relación más fuerte entre estos dos constructos, a diferencia de los de 10 y 11 años. Posiblemente, estas diferencias en la intensidad de la relación que se observan en los niños de 9 años se relacionan con el desarrollo evolutivo de la moralidad y del pensamiento, propios de esta edad. En esa línea, y en torno a la vinculación entre la edad y la Oposición a la igualdad, Delval (2007) plantea que entre los 10 y 11 años, los niños ven a la sociedad como un lugar sin conflictos y en donde cada elemento de la sociedad funciona a la perfección. A su vez, pueden percibir la pobreza, la desigualdad, aunque al no encajar bien en el esquema anterior, ellos las ven como situaciones excepcionales. Quizás, en las edades estudiadas todavía no existe plena conciencia del concepto de desigualdad a un nivel más realista y todavía tengan la representación del mundo social armónico y a partir del cual difícilmente puedan pensar algún tipo de modificación. En esa línea, algunos autores (Enesco et al., 1995) plantean que el pensamiento a menor edad es menos permeable a las condiciones de su medio social, por lo cual no es plausible que existan diferencias entre sujetos de distintos medios. En cambio estas diferencias pueden aparecer a medida que los sujetos van creciendo.

Por otra parte, con respecto a la dimensión Conservación y la variable sociodemográfica edad, se puede pensar que la relación se da de manera positiva y más fuerte a los 9 años debido a que según Delval (2007) en ese primer nivel que se extiende hasta los 10 - 11 años (aproximadamente), los niños se apropian y entienden el mundo social a partir de los aspectos más visibles o perceptivos, sin tener en cuenta procesos ocultos que deban ser inferidos. En el caso de la norma, el niño recibe valores, normas transmitidas por el ambiente, pero no las reproduce intactas, sino que parece integrarlas dentro de su concepción del mundo según el nivel cognitivo alcanzado a su edad. Es de- 
cir que cuando son más pequeños, los niños aceptan y reproducen las ideas y costumbres tradicionales pero debido a su nivel cognitivo, no pueden imaginarse un reajuste de las mismas, ni otra alternativa. En relación a esto, Tap y Kholberg (1971) en un estudio en el que indagaban sobre qué es la norma, por qué la cumplimos y si es modificable, en niños y jóvenes, encontraron que los más pequeños tienen una mirada más restrictiva $\mathrm{e}$ inmutable de la norma, al mismo tiempo que los individuos mayores aceptan que son modificables y justifican la obediencia a ciertas reglas por una convicción interior de justicia.

Asimismo, se hallaron algunas diferencias en la relación entre la Oposición a la igualdad y la Conservación en función de la variable sociodemográfica sexo de los participantes, en tanto la vinculación entre estos dos constructos se muestra más fuerte entre los varones que entre las niñas. Estos resultados quizás tienen que ver con la socialización en roles de género y las expectativas sociales pautadas para cada género.

Por otra parte, en lo que respecta a las vinculaciones entre el Autoritarismo, la Orientación a la dominancia social y los valores sociales con las variables sociodemográficas (edad y sexo de los participantes), no se han encontrado asociaciones significativas entre el sexo y las variables en estudio en los análisis efectuados. Estos resultados coinciden con una línea de estudios previos que tampoco han dado cuenta de la invarianza de género en las variables mencionadas (Castro Solano \& Nader, 2006; Comuni, Langelotti, Jaume, Rodriguez \& Etchezahar, 2010; Imhoff \& Brussino, 2011a; Petit \& Costa, 2011; Roccato, 1997; Roccato et al., 2000; Seoane \& Garzón, 1992; Sosa \& Zubieta, 2008; Zubieta et al., 2007). Estos resultados tal vez podrían vincularse con el hecho de que los niños aún se encuentran en pleno proceso de desarrollo y construcción de su personalidad y por tanto, tienen menos interiorizados los roles de género en comparación con los adultos.

En lo concerniente a la relación entre las variables en estudio y la edad de los partici- pantes, no se observó una relación estadísticamente significativa entre la sub-dimensión Sumisión autoritaria y dicha variable sociodemográfica. Estos resultados coinciden con la mayoría de los estudios revisados (Comuni et al., 2010; Imhoff \& Brussino, 2011a; Petit \& Costa, 2011; Roccato, 1997; Roccato et al., 2000; Seoane \& Garzón, 1992). No obstante, y de manera inesperada, se halló que la sub-dimensión Agresión autoritaria y la edad parecen relacionarse significativa e inversamente, lo cual podría estar asociado con el nivel de desarrollo moral propio de esta edad. Así, tal como se mencionó anteriormente, los niños tienden a aferrarse a las normas sociales, aún cuando las mismas están dirigidas a sustentar la agresión autoritaria contra aquellos individuos que las quebranten. Posiblemente, esto se debe a que los niños de este grupo etario aún no han desarrollado plenamente su capacidad crítico-reflexiva que les permita realizar un cuestionamiento de dichas normas, logro que será alcanzado con la maduración de la edad y la gradual complejización de su nivel cognitivo. No obstante, es preciso destacar que si bien este resultado refuta nuestra hipótesis, la relación encontrada fue débil, lo que podría indicar la necesidad de volver a estudiar y corroborar esta relación.

En lo que refiere a la correlación entre ODS y la edad de los niños, inesperadamente se encontró que tal variable sociodemográfica se relaciona inversa y significativamente tanto con la Oposición a la igualdad como con la Orientación a la dominancia grupal. Quizás, sería necesario analizar nuevamente esta relación a partir de un estudio longitudinal, en el que sea posible observar el nivel de Orientación a la dominancia social en el niño durante su desarrollo. Además, como se mencionó anteriormente, quizás dichos resultados estarían vinculados con la internalización de la norma moral, es decir, aquello que las distintas instituciones transmiten acerca de lo que está bien o mal. De este modo, se piensa que sería interesante efectuar un estudio en el que sea posible analizar los procesos de socialización política, el rol de las agencias socializadoras y su 
relación con el impacto de dichas experiencias en el desarrollo moral de los niños.

Por último, en lo que respecta a la relación entre los valores sociales y la edad de los participantes, inesperadamente sólo se obtuvo una correlación significativa y positiva, aunque débil entre la sub-dimensión Autotrascendencia y la edad, datos que refutan nuestra hipótesis. Es posible que estos resultados se puedan asociar, como ya se mencionó, con la adhesión de los niños a determinados valores aún caracterizados por la inestabilidad, debido al hecho de que todavía se encuentran en pleno proceso de desarro1lo. No obstante, en tanto este último sea enmarcado en procesos de socialización en los que se favorezcan y se refuercen vínculos de colaboración y solidaridad, es posible que en un futuro los niños puedan estar mayormente orientados a priorizar valores tales como la benevolencia y el universalismo. Por otra parte, no se obtuvieron vinculaciones significativas entre el resto de las sub-dimensiones de los valores con la edad, lo cual coincide con los resultados obtenidos en otros estudios (Bubeck \& Bilsky, 2004; Castro Solano \& Nader, 2006; Zubieta et al., 2007) y confirman nuestra hipótesis de trabajo acerca de esta relación.

Para resumir, si bien algunas de las relaciones y asociaciones encontradas no resultaron esperables, es posible trazar algunas tendencias entre las variables estudiadas que podrían estar sugiriendo ciertos perfiles. Por una parte se encontró que una alta Agresión autoritaria va acompañada de una alta Orientación a la dominancia grupal, y que estas dimensiones parecen sustentarse en valores sociales de Conservación. A su vez, una alta Orientación a la dominancia grupal se relaciona con una preferencia por valores de $\mathrm{Au}$ topromoción y con un bajo nivel de adhesión a valores de Autotrascendencia. Estos aspectos podrían ser indicios de la temprana conformación de lo que Cárdenas y colaboradores (2010) y Rottenbacher de Rojas (2010) denominan ideología conservadora. Por otra parte, un perfil diferencial parece ser el de aquellos niños que, adhiriendo a valores de Apertura al cambio y Autotrascendencia, manifiestan niveles más bajos de Oposición a la igualdad. En esa línea, sería interesante realizar futuros estudios a los fines de lograr un mayor esclarecimiento acerca de la presencia de estos posibles perfiles.

Antes de finalizar, es preciso señalar que si bien el trabajo que se informa constituye un primer acercamiento al estudio del autoritarismo en la infancia y su relación con otras variables de corte psicosocial y psicopolítico, los resultados obtenidos no pueden ser generalizados debido al muestreo utilizado. Esta limitación señala la importancia de profundizar en las distintas relaciones y dinámicas de estos constructos en la niñez a través de un muestreo probabilístico. 
Marasca, M., Marasca, R. e Imhoff

TABLA 1

VINCULACIONES ENTRE EL AUTORITARISMO CON LA ORIENTACIÓN DE DOMINANCIA SOCIAL Y VALORES SOCIALES

\begin{tabular}{|l|c|c|}
\hline & Sumisión autoritaria & Agresión autoritaria \\
\hline Orientación de dominancia grupal & .069 & $.110^{*}$ \\
Oposición a la igualdad & $-.146^{* *}$ & .099 \\
Valores de Autotrascendencia & .057 & -.019 \\
Valores de Autopromoción & .021 & .063 \\
Valores de Conservación & $.375^{\star *}$ & $.129^{\star}$ \\
Valores de Apertura al cambio & $.104^{\star}$ & -.009 \\
\hline
\end{tabular}

${ }^{* *}$ La correlación $r$ de Pearson es significativa al nivel de .01

*La correlación $r$ de Pearson es significativa al nivel de .05

\section{TABLA 2}

RELACIONES ENTRE LA ORIENTACIÓN DE DOMINANCIA SOCIAL Y LOS VALORES SOCIALES

\begin{tabular}{|l|c|c|}
\hline & Orientación de dominancia grupal & Oposición a la igualdad \\
\hline Valores de Autotrascendencia & $-.171^{* *}$ & $-.235^{\star *}$ \\
Valores de Autopromoción & $.258^{\star *}$ & -.058 \\
Valores de Conservación & $.111^{*}$ & $-.235^{\star *}$ \\
Valores de Apertura al cambio & -.019 & $-.129^{*}$ \\
\hline
\end{tabular}

** La correlación $r$ de Pearson es significativa al nivel de .01

* La correlación $r$ de Pearson es significativa al nivel de .05 


\section{REFERENCIAS BIBLIOGRÁFICAS}

Adorno, T., Frenkel-Brunswuik, E., Levinson, D. \& Sanford, R. (1950). La personalidad autoritaria [The authoritarian personality]. Buenos Aires: Editorial Proyección.

Altemeyer, B. (1988). Enemies of freedom: Understanding Right-Wing authoritarianism. San Francisco: Jossey-Bass.

Altemeyer, B. (1993). Nacionalismo y autoritarismo de derechas entre legisladores americanos [Nationalism and right wing authoritarianism between American legislators]. Psicología Política, 7, 7-18.

Altemeyer, B. (1998). The other "Authoritarian Personality". Advances in Experimental Social Psychology, 30, 47-92. doi: 10.1016/S006526 01(08)60382-2.

Altemeyer, B. (2004). The other "authoritarian personality”. En J.T. Jost \& J. Sidanius (Eds.), Political Psychology (pp. 85-107). New York: Psychology Press.

Altemeyer, B. (2006). The authoritarians. Canadá: University of Manitoba. Winnipeg.

Arce, S., Cordera, M.E. \& Perticarari, M. (2010). La construcción de la prosocialidad en niños y adolescentes en la ciudad de Córdoba [Prosociality's construction in children and teenagers in Córdoba city]. Proyecto de investigación, SECYT-UNC. Argentina.

Benedicto, J. \& Morán, M. (1995). La construcción de los universos políticos de los ciudadanos [The construction of citizens' political universes]. En J. Benedicto \& M. Morán (Eds.), Sociedad y politica. Temas de sociología política (pp. 227-258). Madrid: Alianza.

Boehnke, K. \& Rippl, S. (1995). ¿Produce autoritarismo el socialismo? Una comparación de los jóvenes de Alemania Oriental con Alemania Occidental y EE.UU [Does socialism produce authoritarianism? A comparison of East German with West Germany and the U.S young people]. Psicología Política, 10, 87105.

Bubeck, M. \& Bilsky, W. (2004). Value structure at an early age. Swiss Journal of Psychology,
1(63), 31-41. doi: 10.1024/1421-0185.63.1. 31.

Cárdenas, M., Meza, P., Lagues, K. \& Yañez, S. (2010). Adaptación y validación de la Escala de Orientación a la Dominancia Social (SDO) en una muestra chilena [Adaptation and validation of Social Dominance Orientation's Scale (SDO) in a Chilean sample]. Universitas Psicológica, 9(1), 161-168.

Cárdenas, M. \& Parra, L. (2010). Adaptación y validación de la Versión Abreviada de la Escala de Autoritarismo de Derechas (RWA) en una muestra chilena [Adaptation and validation of Right Wing's Authoritarism Scale (RWA) abridged version in Chilean sample]. Revista de Psicología, 19(1), 61-79.

Castro Solano, A. \& Nader, M. (2006). La evaluación de los valores humanos con el Portrait Values Questionnaire de Schwartz [The evaluation of human values with Schwartz's Portrait Values Questionnaire]. Interdisciplinaria, 23 (2), 155-174.

Chatard, A. \& Selimbegovic, L. (2008). The intergenerational transmission of social dominance: A three-generation study. European Journal of Personality, 22, 541-551. doi: 10.1002/per.684.

Cima, R. \& Dallago, F. (2007). Existe una correlación negativa entre el autoritarismo de derechas y la orientación a la dominancia social [There's a negative interrelationship between the Right Wing Authoritarianism and the social dominance orientation]. Psicologia Política, 34, 79-97.

Cohrs, J.C., Moschner, B., Maes, J. \& Kielmann, S. (2005). The motivational bases of RightWing Authoritarianism and social dominance orientation: Relations to values and attitudes in the after math of September 11, 2001. Personality and Social Psychology Bulletin, 31(10), 1425-1434.

Comuni, A., Langelotti, L., Jaume, L.C., Rodriguez, F.A. \& Etchezahar, E. (2010). Autoritarismo de derechas y posicionamiento ideológico en estudiantes universitarios [RightWing authoritarianism and ideological positioning in university students]. Memorias del 
II Congreso Internacional de Investigación y Práctica Profesional en Psicología. XVII Jornadas de Investigación. Sexto Encuentro de Investigadores en Psicología del MERCOSUR (Tomo IV, pp. 73-75). Facultad de Psicología. Universidad Nacional de Buenos Aires, Argentina.

D’Adamo, O. \& García Beaudox, V. (1996). Creencias sociales contemporáneas y sistema democrático [Contemporary social beliefs and democratic system]. Psicología Política, 12, $35-45$.

Delgado Rodríguez, M. \& Llorca Díaz, J. (2004). Estudios longitudinales: Concepto y particularidades [Longitudinal studies: Concept and particularities]. Revista Española de Salud Pública, 78(2), 141-148.

Delval, J. (2007). Aspectos de la construcción del conocimiento sobre la sociedad [Aspects of knowledge's construction about society]. Revista de Investigación en Psicología, 10(1), 948. doi: 10.1590/S0104-40602007000200004.

Duckitt, J. (2001). A dual-process cognitive-motivational theory of ideology and prejudice. $A d-$ vances in Experimental Social Psychology, 33, 41-113. doi: 10.1016/S0065-2601(01)800 046.

Duriez, B., Soenens, B. \& Vansteenkiste, M. (2007). In search of the antecedents of adolescent authoritarianism: The relative contribution of Parental Goal Promotion and Parenting Style Dimensions. European Journal of Personality, 21, 507-527. doi: 10.1002/per. 623.

Enesco, I., Delval, J., Navarro, A., Villuendas, D., Sierras, P. \& Peñaranda, A. (1995). La comprensión de la organización social en niños y adolescentes [The understanding of social organization in children and teenargers]. $\mathrm{Co}$ lección Investigación, 104. Editorial Secretaría General Técnica. Universidad Autónoma de Madrid.

Espinoza, A. \& Calderón-Prada, A. (2006). Ideología política, valores culturales y miedo a la muerte. Su impacto después de los atentados del 11 de marzo [Political ideology, cultural values and scary to death. Their impact after the 11th March attempt]. Psicología Política, 32, 33-58.

Espinosa A., Calderón-Prada, A., Burga, G. \& Güímac, J. (2007). Estereotipos, prejuicios y exclusión social en un país multiétnico: El caso peruano [Stereotypes, prejudgements and social exclusion in a multiethnic country: The Peruvian case]. Revista de Psicología, 25(2), 295-338.

Etchezahar, E. (Noviembre, 2011). Dimensionalidad de la Escala de Autoritarismo del ala de Derechas (RWA) [The dimensionality of Right Wing Authoritarianism's scale (RWA)]. Actas del I Encuentro Ibero-Latinoamericano de Grupos y Equipos de Psicología Política. Facultad de Psicología. Universidad Nacional de Córdoba, Argentina.

García-Castro, J.D. (2010). Ideología de la desigualdad: Análisis de la investigación empírica en psicología social [Inequality's ideology: Analysis of the empirical research in social psychology]. Revista Electrónica de Psicología Política, 24, 67-87.

Giovanni Di Stefano, G.F. (2005). Le relazioni tra autoritarismo di destra, orientamento alla dominanza sociale e struttura psicologica universale dei valori di Schwartz [The relationship between the Right Wing Authoritarianism, the social dominance orientation and the universal psychological structure of Schwartz's values]. Convegno Il valore dei valori. Sessione Poster. Università Degli Studi di Catania. Università Kore' di Enna.

Hair, J.F., Anderson, R.E., Tatham, R.L. \& Black, W.C. (1999). Análisis multivariante [Multivariate analysis]. Madrid: Prentice Hall.

Hernández Sampieri, R., Fernández Collado C. \& Baptista, P. (2003). Metodología de la investigación [Methodology of research], (3ra. ed.). México: Mc Graw Hill.

Huerta, E., Bañuelos, B., Rodríguez, A., Luz, S. \& Gómez, C. (2006). El rol de la televisión en la socialización política de los niños: Resultados preliminares [The television role in the children's po- 
litical socialization: Preliminary results]. UNIrevista, 1(3), 1-12.

Imhoff, D. \& Brussino, S. (2010). Estudio cuasiexperimental acerca de la influencia de una experiencia de socialización política alternativa sobre el desarrollo moral, los valores, competencias y nociones socio-políticas de niñas y niños cordobeses [A quasi-experimental study relating to the influence of an alternative political socialization experience about moral development, the values, competences and sociopolitical notions of girls and boys from Córdoba]. Proyecto doctoral. Facultad de Psicología, Universidad Nacional de Córdoba. Argentina.

Imhoff, D. \& Brussino, S. (2011a). Reflexiones acerca del proceso de socialización política infantil a partir de la exploración del autoritarismo en niñas y niños cordobeses [Reflections about the children's political socialization process from the exploration of the authoritarianism in girls and boys from Córdoba]. Actas del X Congreso Nacional de Análisis Político (pp. 1-23). Córdoba, Argentina.

Imhoff, D. \& Brussino, S. (2011b). Portrait Values Questionnaire: Exploración en torno a sus propiedades psicométricas en el contexto de Córdoba / Argentina [Portrait Values Questionnaire: Exploration towards its psychometric properties in the Córdoba / Argentina's context]. Manuscrito no publicado.

Imhoff, D. \& Brussino, S. (2012). Análisis de las propiedades psicométricas de la Escala ODS con niños cordobeses [Analysis of SDO Scale's psychometric properties with children from Córdoba]. Manuscrito no publicado.

Jiménez Burillo, F., Del Águila Tejerina, R., Luque, E., Sangrador García, J.L. \& Vallespín Oña, F. (2006). La personalidad autoritaria [The authoritarian personality]. Psicología de las relaciones de autoridad y de poder (Cap. 5, pp. 127-168). España: Editorial UOC.

Knafo, A. (2003). Authoritarians, the next generation: Values and bullying among adolescent children of authoritarian fathers.
Analyses of Social Issues and Public Policy, 3(1), 1-6.

Lacerda Teixeira Pires, A.M. (2010). El prejuicio racial en Brasil: Medidas comparativas [The racial prejudice in Brazil: Comparative measures]. Psicología \& Sociedade, 1(22), 32-42.

Lemos, V. (2006). La deseabilidad social en la evaluación de la personalidad infantil [The social desirability in the evaluation of children's personality]. Suma Psicológica, 13, 7-14.

Llinares Insa, L., Molpeceres Pastor, Ma. A. \& Musitu, G. (2001). La autoestima y las prioridades personales de valor. Un análisis de sus interrelaciones en la adolescencia [The selfsteem and value's personal priorities. An analysis of their interrelationships in adolescence]. Revista Anales de Psicología, 17(2), 189-200.

Mendoza, R. (2010). Control social subjetivo, valores, creencias y actitudes politicas: El efecto oveja negra. Un estudio experimental transcultural en Iberoamérica [Subjective social control, values, beliefs and political attitudes: the black sheep effect. A cross-cultural experimental study in Latin America]. Tesis Doctoral. Facultad de Psicología. Universidad del País Vasco. San Sebastián. España. Recuperado el 13 de diciembre de 2011 de https:// www. educacion.gob.es/teseo/imprimirFicheroTesis. do?fichero $=19827$.

Mischel, W., Zeiss, R. \& Zeiss, A. (1974). Internal-external control and persistence: Validation and implications on The Stanford Preschool Internal-External Scale. Journal of Personality and Social Psychology, 29, 265278. doi: 10.1037/h0036020.

Montes Berges, B. \& Rodríguez García, C. (2006). La dominancia en el continuo personal-grupal: Dominancia personal, interpersonal y SDO [The dominance in the personal - group continuum: Personal dominance, interpersonal and $\mathrm{SDO}$ ]. Revista Electrónica Iniciación a la Investigación, 1(18), 1-7.

Ortiz Zabala, M. (1985). Relaciones empíricas entre personalidad, autoritarismo y valores 
[Empirical relationships between personality, authoritarianism and values]. Murcia: Editorial Hijos de E. Minuesa.

Petit, L. \& Costa, G.L. (2011). Dominancia social: El género como jerarquía social [Social dominance: The gender as social hierarchy]. Hologramática, 3(14), 71-83.

Petit, L., López, A.L. \& Sánchez, P. I. (2010). Orientación a la Dominancia Social (SDO) en estudiantes de la Facultad de Psicología de la UBA: Invarianza de género como componente de las estructuras jerárquicas [Social Dominance Orientation (SDO) in students from Psychology's Faculty (UBA): Gender's invariance as a component of hierarchical structures]. Memorias del II Congreso Internacional de Investigación y Práctica Profesional en Psicología. XVII Jornadas de Investigación. Sexto Encuentro de Investigadores en Psicología del MERCOSUR (Tomo IV, pp. 159-161). Facultad de Psicología. Universidad Nacional de Buenos Aires, Argentina.

Pettigrew, T.F. \& Meertens, R.W. (1995). Subtle and blatant prejudice in Western Europe. European Journal of Social Psychology, 25, 57-75.

Roccato, M. (1997). Autoritarismo de derechas y adolescencia [Right wing authoritarianism and adolescence]. Psicología Política, 14, 61-76.

Roccato, M., Gattino, S. \& Patris, E. (2000). Personalidad, valores y orientación política [Personality, values and political orientation]. Psicología Politica, 21, 73-97.

Rottenbacher de Rojas, J. (2010). Sexismo ambivalente, paternalismo masculino e ideología política en adultos jóvenes de la ciudad de Lima [Ambivalent sexism, masculine paternalism and political ideology in young adults from Lima city]. Revista Pensamiento Psicológico, 7(14), 9-18.

Rottenbacher de Rojas, J., Amaya López, L., Genna Miyahira, K. \& Pulache Páez, M. (2009). Percepción de inseguridad ciudadana y su relación con la ideología política en una muestra de habitantes de la ciudad de Lima
[Public insecurity's perception and its relationship with the political ideology in a sample of residents from Lima city]. Revista Española de Investigación Criminológica, 7, 1-22.

Schwartz, S. \& Rubel-Lifschitz, T. (2009). Crossnational variation in the size of sex differences in values: Effects of gender equality. Journal of Personality and Social Psychology, 97(1), 171-185. doi: 10.1037/a0015546.

Seoane, J. \& Garzón, A. (1992). Creencias sociales contemporáneas, autoritarismo y humanismo [Social contemporary beliefs, authoritarianism and humanism]. Psicología Politi$c a, 5,27-52$.

Sidanius, J. \& Pratto, F. (1999). Social dominance: An intergroup theory of social hierarchy and oppression. New York: Cambridge University Press. doi: 10.1017/CBO9781139 175043.

Sidanius, J., Pratto, F., Van Laar, C. \& Levin, S. (2004). Social dominance theory: Its agenda and method. Political Psychology, 25, 845880. doi: 10.1111/j.1467-9221.2004.00401.x.

Silván-Ferrero, M. \& Bustillos, A. (2007). Adaptación de la Escala de Orientación a la Dominancia Social al castellano: Validación de la dominancia grupal y la oposición a la igualdad como factores subyacentes [Adaptation of the Social Dominance Orientation's Scale to Spanish: Validation of the group-based dominance and opossition to equality as underlying factors]. Revista Fundación Infancia y Aprendizaje, 1(22), 3-15. doi: 10.1174/0213474077 79697485.

Sosa, F.M. \& Zubieta, E.M (2008). Valores y multiculturalismo [Values and multiculturalism]. Memorias de las XV Jornadas de Investigación. Cuarto Encuentro de Investigadores en Psicología del MERCOSUR. Problemáticas actuales. Aportes de la investigación en Psicología (Tomo III, pp. 506-507). Facultad de Psicología. Universidad Nacional de Buenos Aires, Argentina.

Tapp. J. \& Kholberg, K. (1971). Developing senses of law and legal justice. Journal of So- 
cial Issues, 27, 65-91. doi: 10.1111/j.154 0-45 60.1971.tb00654.x.

Van Hiel, A., Pandelaere, M. \& Duriez, B. (2004). The impact of need for closure on conservative beliefs and racism: Differential mediation by authoritarian submission and authoritarian dominance. Personality and Social Psychology Bulletin, 30, 824-837. doi: 10.1177/0146 167204264333.

Zaiter, J. (2002). Psicología social del autoritarismo [Authoritarianism's social psychology]. Revista Perspectivas Psicológicas, 2, 37-48.
Zubieta, E. \& Delfino, G. (Noviembre, 2011). Autoritarismo, dominancia social y valores sociales [Authoritarianism, social dominance and social values]. Actas del I Encuentro Ibero-Latinoamericano de Grupos y Equipos de Psicología Política. Facultad de Psicología. Universidad Nacional de Córdoba. Argentina. Zubieta, E., Delfino, G. \& Fernández, O. (2007). Dominancia social, valores y posicionamiento ideológico [Social dominance, values and ideological positioning]. Revista Psicodebate, 8. Psicología, Cultura y Sociedad, 151-170.

\author{
Equipo de Psicología Politica \\ Laboratorio de Psicología Cognitiva \\ Facultad de Psicología \\ Universidad Nacional de Córdoba (UNC) \\ Córdoba \\ República Argentina
}

Fecha de recepción: 2 de noviembre de 2012 Fecha de aceptación: 10 de junio de 2013 


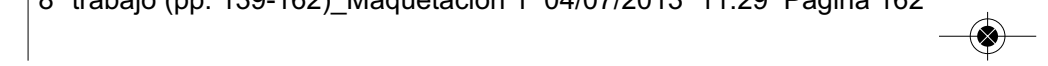

